\title{
Failure mode transitions of unconfined granular media from dry to unsaturated to "quasi-fully" saturated states
}

\author{
Katherine Winters ${ }^{1, *}$, Woodman Berry ${ }^{1}$, Greg Seimens ${ }^{2}$, and Oliver-Denzil Taylor ${ }^{1}$ \\ ${ }^{1}$ U.S. Army Corps of Engineers, Engineer Research and Development Center, Geotechnical and Structures Laboratory, 3909 Halls Ferry \\ Rd, Vicksburg, MS, USA \\ ${ }^{2}$ GeoEngineering Centre at Queen's-RMC, 13 General Crerar, Sawyer Building, Room S3418, Department of Civil Engineering, Royal \\ Military College of Canada, Kingston, ON, Canada
}

\begin{abstract}
Recent research has shown discrepancies between the prevailing mathematical representations of near-surface shear strength and the observed shear strengths. This investigation focuses on three granular materials, i.e., 1) poorly-graded, medium-fine silica-quartz sand, 2) an engineered silica-quartz mix of 3.38$\mathrm{mm}$ and $0.638-\mathrm{mm}$ sub-angular particles, and 3) an angular fused quartz sand. Specimens were tested under load-controlled conditions at variable saturations in order to identify and quantify the influence of suction on the granular structures and failure modes. All three materials exhibited localized radial particle force chain buckling failures in unconfined drained dry (UDK) conditions and classical shear failures in the unconfined drained unsaturated shear (UDP) conditions. In unconfined drained suction failures (UDS) conditions, the poorly-graded, medium-fine silica-quartz sand exhibited a bulging and sloughing failure without weeping, while the other two materials wept and then held loads before failure. Thus, it is suggested that the pore fluid had a predominate lubrication (strength weakening) effect, and the assumption of structure stiffening (strength increase) from matric suction may not be valid at near-surface conditions for sub-angular silica-quartz materials but is valid for the angular fused quartz.
\end{abstract}

\section{Introduction}

Understanding the true behavior of cohesionless soils immediately impacts the fields of internal erosion prediction, surficial sloughing in dams and levees, wave propagation, geo-sensor coupling, and geoenvironmental contamination and remediation designs. Laboratory experimentation on cohesionless soil fabric behavior in zero-to-low vertical confining pressure environments relies on the use of effective stress principles to infer behavior [1-3]. However, research suggests that the use of effective stress principles is not indicative of nearsurface environments due to the application of confining pressures to maintain sample stability of granular materials. Moreover, recent research has shown discrepancies between the prevailing mathematical representations of near-surface shear strength and the observed shear strengths, e.g., Equation 1. This classical approach has proved sufficient for soils that are not at, or in close proximity to, the elastic free boundary of the soil-atmosphere interface.

$$
\tau=c^{\prime}+\sigma^{\prime}{ }_{\mathrm{n}} \tan \left(\phi^{\prime}\right)+s S_{r}^{\xi} \tan \left(\phi^{\prime}\right)
$$

where $\sigma_{n}$ is the normal effective stress along the failure plane, $c^{\prime}$ is the soil cohesion, $\phi^{\prime}$ is the internal friction angle, and the $s S_{r}^{\xi}$ parameter is used to account for the non-linearity suction strength from the soil-water characteristic curve (SWCC) that provides for additional soil matrix strength in unsaturated soil mechanics [4]. More importantly, the mathematical representations require the assumption that the onset of failure is continuous along a single rupture plane at an angle, $\alpha$, from horizontal to experimentally define $\phi^{\prime}$, as in Equation 2 [5].

$$
\alpha=45^{\circ}+\frac{\phi^{\prime}}{2}
$$

Taylor et al. [6] showed that the failure modes of unconstrained (free-standing) physical granular specimens do not conform to the assumption of simultaneous failure, especially under dry conditions. The purpose of this paper is to investigate the failure transitional modes observed in Taylor et al. [6] with respect to changes in particle size and shape, i.e., subangular and platey materials at different degrees of saturation.

\section{Materials}

This investigation focuses on three different granular materials, i.e., 1) poorly-graded, medium-fine silicaquartz beach sand (abbreviated here as S101), 2) an engineered silica-quartz mix of 3.38-mm and 0.638-mm sub-angular particles (E57-60), and 3) an angular fused quartz sand that is a refined slag byproduct with a platey 
crystalline structure (T101). Previous research on these materials can be found in several places in the literature [6-12]. None of the materials contained any fines or gravels. Engineering properties of the three materials can be found in Table 1, and the grain size distributions are provided in Figure 1.

Table 1. Material Properties.

\begin{tabular}{|c|c|c|c|c|}
\hline Material & 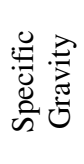 & 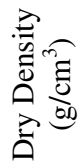 & 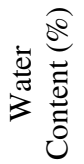 & 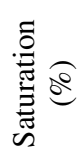 \\
\hline $\begin{array}{l}\text { Poorly Graded } \\
\text { Sand (S101) }\end{array}$ & 2.67 & 1.69 & 5.32 & 24 \\
\hline $\begin{array}{l}\text { Engineered Mix } \\
\text { (E57-60) }\end{array}$ & 2.62 & 1.82 & 6.09 & 36 \\
\hline $\begin{array}{l}\text { Fused Quartz } \\
\quad(\mathrm{T} 101)\end{array}$ & $\begin{array}{c}2.24 \\
{[6]}\end{array}$ & 1.43 & 5.30 & 21 \\
\hline
\end{tabular}

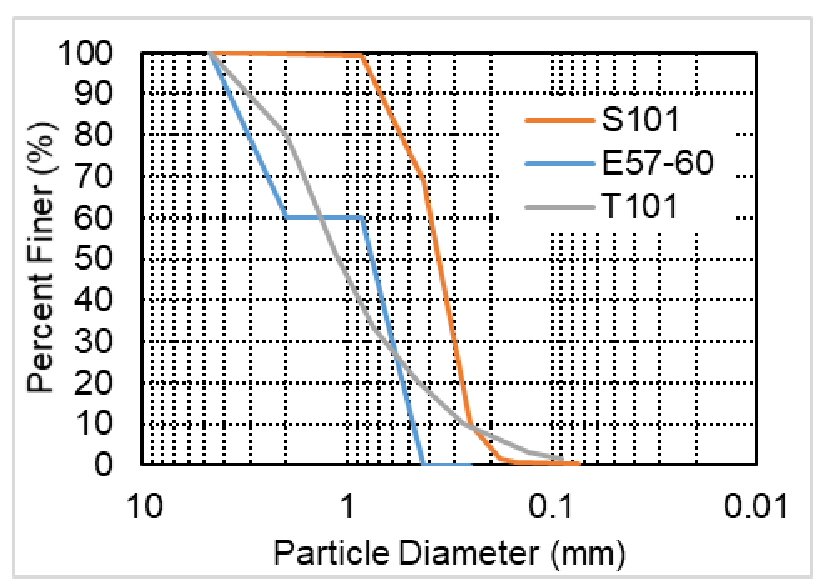

Fig. 1. Grain Size Distribution.

\section{Methods}

Test specimens were prepared via an energy controlled moist tamping method [11] using a 71-mm diameter mold to a height of $145 \mathrm{~mm}$. The applied energy was 600 $\mathrm{kJ} / \mathrm{m}^{3}$, representative of a medium-dense field condition. The specimens were constructed in a standard triaxial mold, which was removed immediately following construction. As in Taylor et al. [6], all specimens are self-supported, unconfined, drained, i.e., no chamber, cell, membrane or apparatus is used to support the sample post reconstitution, at atmospheric conditions such that effective stress and total stress regimes are equivalent.

Unconfined specimens were loaded using increments of calibrated metal weights ranging from $50 \mathrm{~g}$ to $1000 \mathrm{~g}$ placed on top of the sand specimen at 20-second increments until initial failure was observed. Total loading times varied based on the number of weights each specimen held; average total loaded time before failure was approximately three to four minutes. Failures were filmed using high-speed high-resolution videography (two cameras at a 1280x800 dpi resolution and $389 \mathrm{fps}$ ). The final weight before failure and the weight causing failure were recorded. The vertical axial stress applied at failure was calculated using the average of the weight held before failure and the weight causing failure. Testing was conducted in approximately 22 to 24 degree Celsius temperatures and $60 \%$ relative humidity.

\subsection{Unconfined drained dry (UDk)}

$U D \kappa$ specimens were allowed to air-dry in a controlled environment for 48 hours and then carefully loaded with precision weights until failure. Laboratory testing showed that a 48-hour drying period resulted in no measurable moisture content in the samples. After failure, three moisture content samples were taken from the failed sample to ensure dry conditions. All specimens had less than $0.01 \mathrm{~g}$ of water per $50 \mathrm{-g}$ moisture content sample.

\subsection{Unconfined drained unsaturated shear $\left(U D_{P}\right)$}

The $U D_{P}$ test was conducted immediately after specimen reconstitution. The specimen was carefully loaded with precision weights until failure. After failure, three moisture content samples were taken from the center, middle, and top of the failed sample. $U D_{P}$ moisture contents after testing were consistent with the initial mixed material and exhibited moisture uniformity throughout the sample. No drying or fluid flow was observed during the testing.

\subsection{Unconfined drained suction failure (UDs)}

The $U D_{S}$ test was conducted immediately after specimen reconstitution. The top of the specimen was uniformly saturated with distilled de-aired water in 3-mL increments, allowing 20 seconds between each fluid increment for permeation of the water through the entire sample. Saturation efforts were suspended at the onset of deformation. If the observed deformation ceased within 20 seconds, the saturation process was continued in the same incremental manner until additional deformation was observed in the high-resolution videography. If the specimen was observed to weep excess pore fluid, saturation efforts were discontinued, and the specimen was instead loaded to failure using calibrated weights. Wetting times varied by specimen but averaged approximately 12 minutes for poorly graded sand S101, five minutes for engineered mix E5760, and 10 minutes for fused quartz T101. Final saturations were calculated assuming no change in volume.

\section{Results}

Figures 2 through 10 provide six-panel images of representative failures for each soil type and test condition. 


\subsection{Poorly Graded Sand S101}

The S101 poorly graded sand failure can been seen in Figure 2. The specimen did not exhibit any bulging or other deformation before failure. The failure can be seen to propagate from the top down with approximately one third of the specimen failing at a time. Nine specimens failed under an applied axial stress ranging between 2.7 and $7.3 \mathrm{kPa}$, with an average of $5.2 \mathrm{kPa}$.
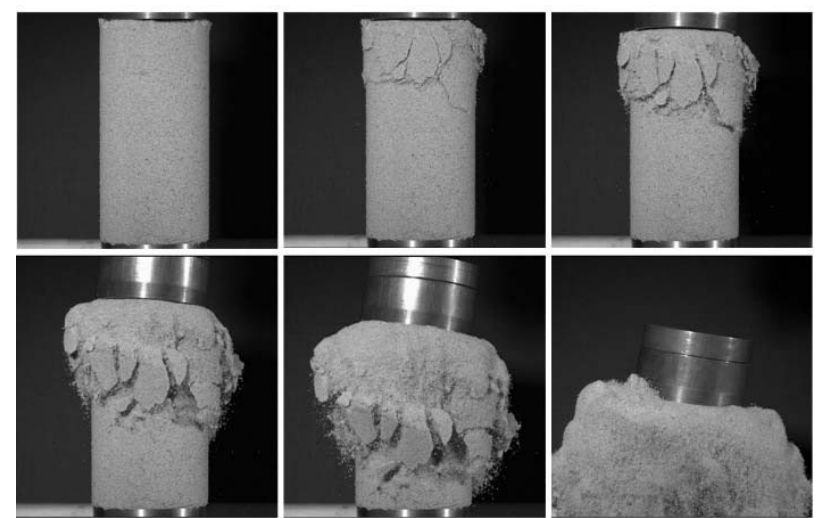

Fig. 2. S101 Dry UDк Failure.

In the wet $U D_{\mathrm{P}}$ specimens in Figure 3, a classical critical state shear plane is visible along with some bulging before failure. These specimens are weaker than the dry UDк specimens with nine specimens failed under a vertical applied stress ranging between 3.7 and $4.6 \mathrm{kPa}$ an average stress of $4.1 \mathrm{kPa}$.
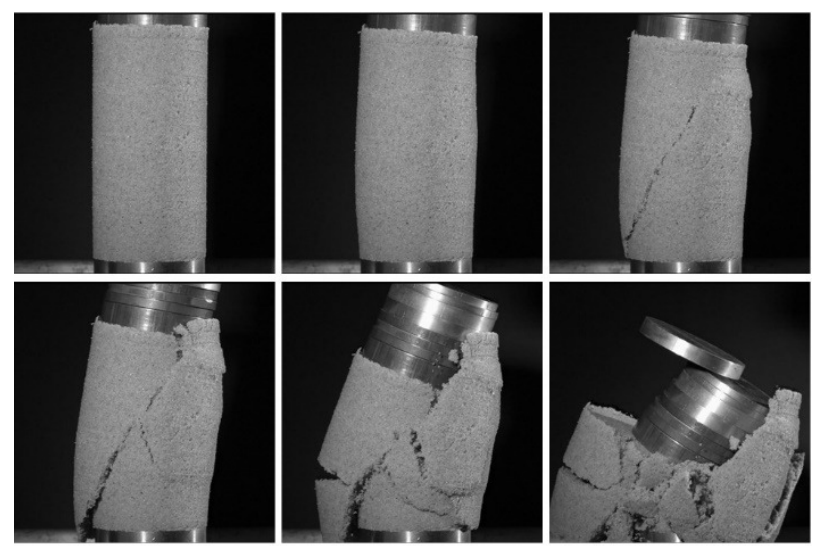

Fig. 3. S101 Wet UD Failure

For the wetting $\mathrm{UD}_{\mathrm{S}}$ test, the nine specimens were wetted until they failed through slumping, as shown in Figure 4. No weights were applied. After failure, three moisture content samples were taken from the failed sample to determine the final moisture content. Specimens failed at an average saturation of $68.8 \%$.
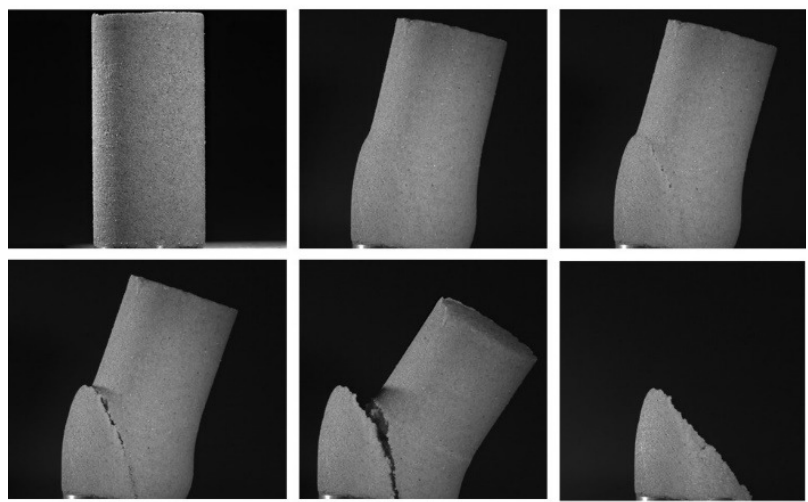

Fig. 4. S101 Wetting $U$ Failure.

\subsection{Engineered Mix E57-60}

The courser engineered mix E57-60 behaved largely the same as the clean sand S101, as can be seen for the UDK specimens in Figure 5. Five UDא specimens failed under a vertical applied stress ranging between 2.7 and $3.4 \mathrm{kPa}$ with an average of $2.9 \mathrm{kPa}$.
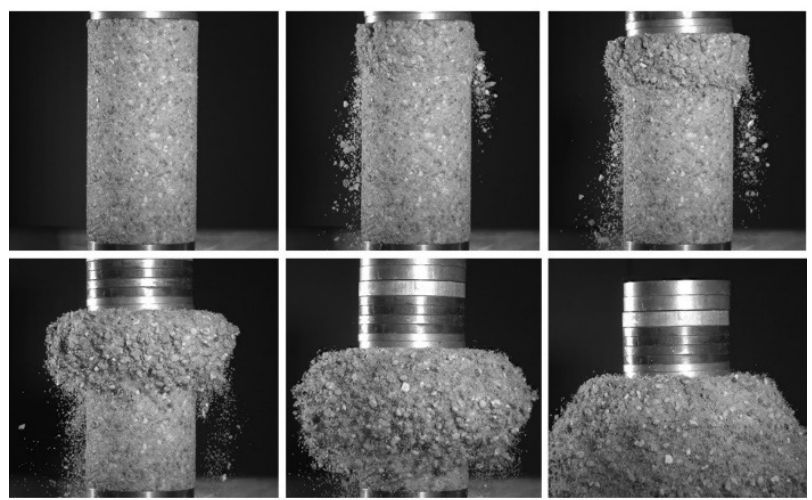

Fig. 5. E57-60 Dry UDк Failure.

E57-60 specimens again behaved similarly to the S101 specimens in $\mathrm{UD}_{\mathrm{P}}$ conditions, although the shear plane is not as pronounced in Figure 6 due to the coarser nature of the material. Eleven specimens failed under a vertical applied stress ranging between 2.7 and $4.6 \mathrm{kPa}$ with an average of $3.3 \mathrm{kPa}$.
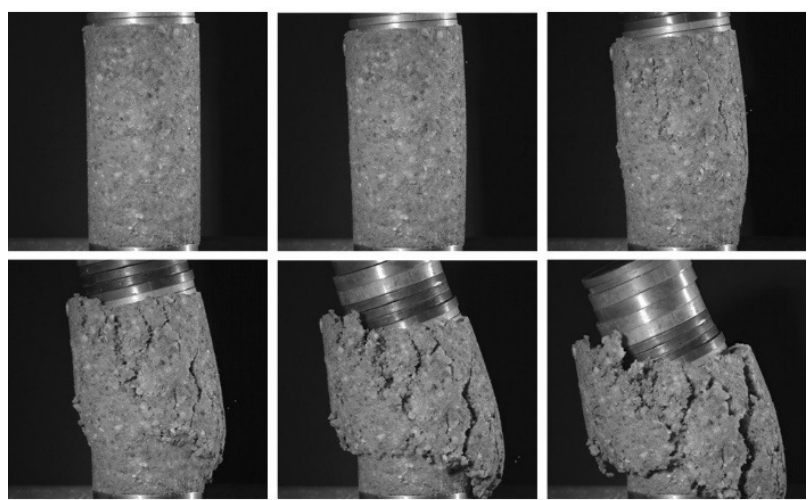

Fig. 6. E57-60 Wet $\mathrm{UD}_{\mathrm{P}}$ Failure. 
The wetting specimens did not fail before the onset of weeping. Light weights were applied as can be seen in Figure 7. The entire specimen was dried to determine the moisture content. Five specimens failed at a saturation of $49 \%$ and under a vertical applied stress ranging between 0.1 and $0.7 \mathrm{kPa}$, with an average of $0.4 \mathrm{kPa}$.
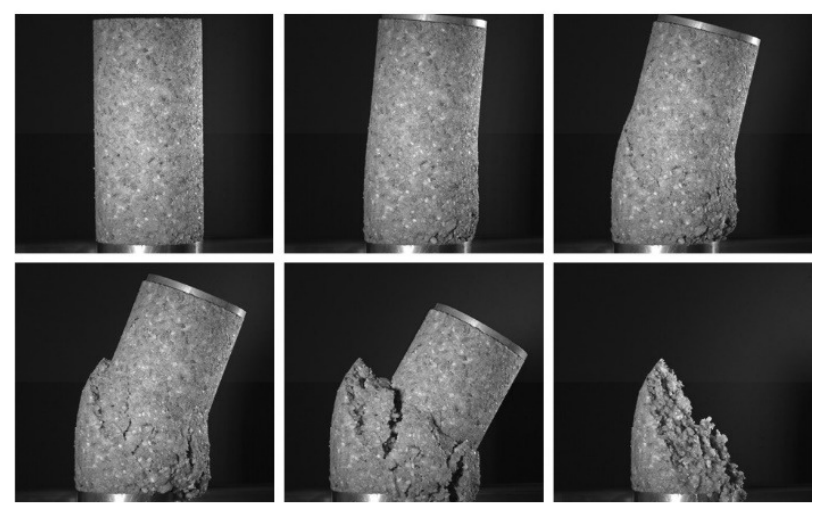

Fig. 7. E57-60 Wetting $U_{S}$ Failure.

\subsection{Fused Quartz T101}

The fused quartz T101 material fabric structure is very fragile when dry. Five UDк specimens initially stood for a minimum of 12 hours but collapsed under their own weight before the standard 48-hour drying period had elapsed. Upon inspection, the specimens were determined to not have reached a fully dry condition. The sixth sample is shown in Figure 9, with a similar failure pattern to the S101 and E57-60 dry specimens. This specimen failed under a $0.2 \mathrm{kPa}$ applied axial stress.
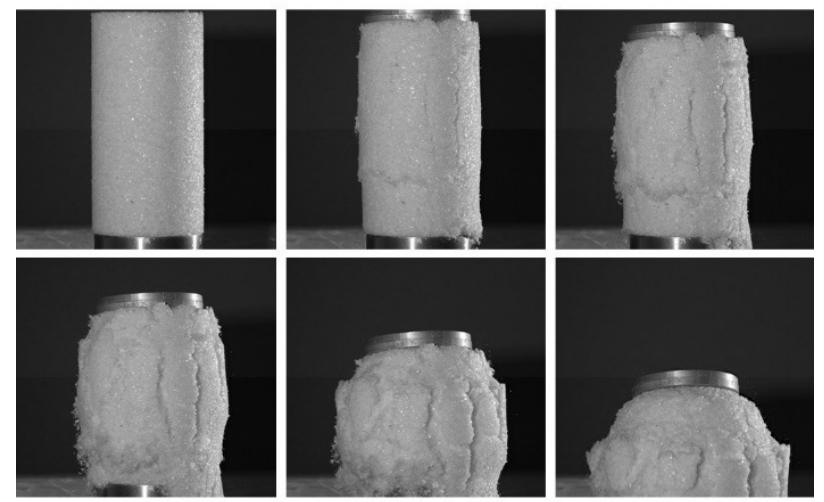

Fig. 8. T101 Dry UDк Failure.

The development of a shear plane can be observed in the failure of the T101 UD $\mathrm{UD}_{\mathrm{P}}$ specimens in Figure 10. However, as the failure progresses, the weights fall straight down instead of following the angle of shear plane, and the material exhibits more of a radial failure. In contrast to the week and fragile UDк specimens, the three $U_{P}$ specimens failed under a vertical applied stress ranging between 13.9 and $16.3 \mathrm{kPa}$ with an average of $15.2 \mathrm{kPa}$, i.e., strengths much higher than those observed for either of the silica-quartz sands.
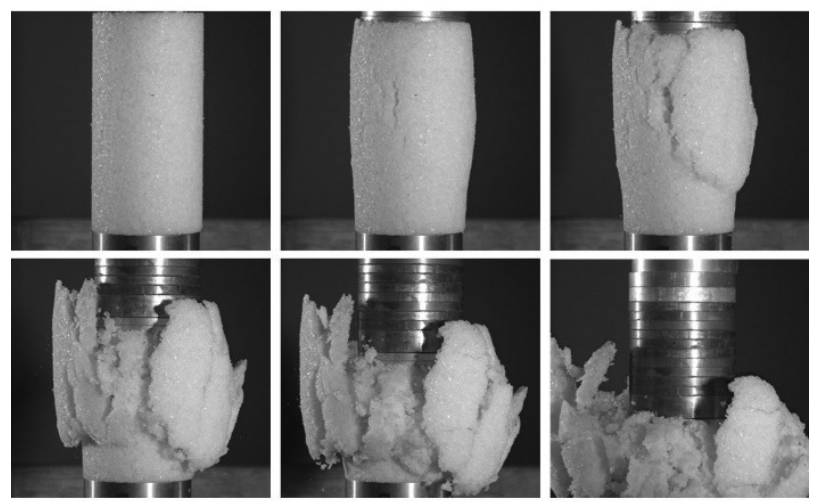

Fig. 9. T101 Wet $U_{P}$ Failure.

The T101 UD specimens did not fail in a consistent manner after the onset of weeping. Of the four specimens, two exhibited a shear failure similar to the classical shear failure of the wet clean sand, as shown in Figure 10. The other specimens were more similar to the $\mathrm{UD}_{\mathrm{P}} \mathrm{T} 101$ samples, with more development of a shear plane combined with radial granular ejecta. The entire specimen was dried to determine the moisture content. Four specimens failed at a saturation of $55 \%$ and under a vertical applied stress ranging between 8.4 and $12.1 \mathrm{kPa}$, with an average of $10.4 \mathrm{kPa}$.
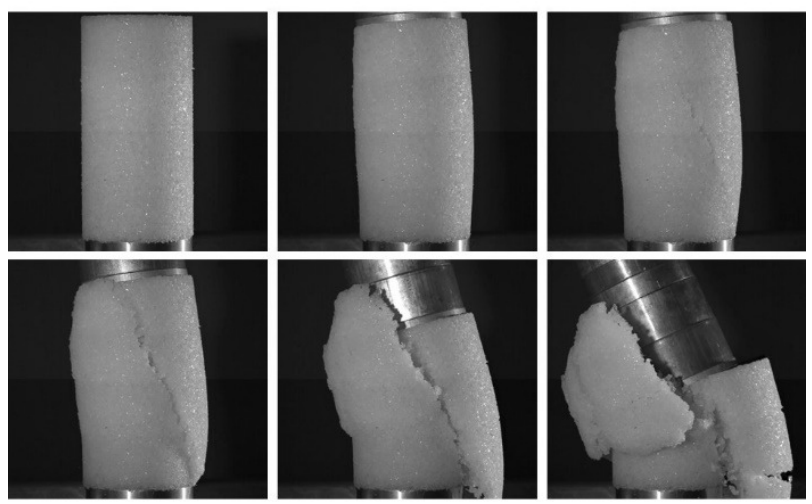

Fig. 10. T101 Wetting $U_{\mathrm{S}}$ Failure.

\section{Discussion}

\subsection{Unconfined drained dry (UDK)}

All three materials exhibited localized radial particle force chain buckling failures in unconfined drained dry $\left(\mathrm{UD}_{\kappa}\right)$ conditions suggesting that failure stress is not uniform throughout the specimen. Pixel matching of high-resolution photographs showed no axial or radial deformation before failure. The failures propagate down from the top of the specimen with radial granular ejecta. In the both the subangular S101 and E57-60 sands, the failure can be seen to begin in the top third of the specimen, while in the platey-shaped T101 material, the failure is in the upper two thirds. The T101 fabric structure is also much weaker and more fragile than the 
others due to less contact friction between the particle plates.

In all cases, the combination of localized buckling failures and no observable deformations prior to the onset of failure suggest that conditions for simultaneous failure is violated. This is further evidenced as Equation 1 suggests that an unconfined dry granular material will not exhibit a shear resistance, which is in contrast with physical experimentation and observations.

\subsection{Unconfined drained unsaturated shear $\left(U D_{P}\right)$}

All three materials exhibited classical shear failures in the unconfined drained unsaturated shear $\left(\mathrm{UD}_{\mathrm{P}}\right)$ conditions. Due to the grain sizes, the critical failure plane is most visible in the S101 material. In the case of these types of failures there is a distinct transition to a simultaneous failure condition due to the inclusion of soil suction in the partially saturated regime. However, this presents an unresolved query of how soil suction should be accounted for in terms of the failure shear stress. Namely, if suction has the effect of generating an effective confining pressure and increasing $\alpha$, then should the effects, in an unconfined state, be counted twice, i.e., once in the increase of the effective friction angle and a second time in the addition of a suction parameter?

\subsection{Unconfined drained suction failure (UDS)}

In unconfined drained suction failure $\left(\mathrm{UD}_{\mathrm{S}}\right)$ conditions, the poorly-graded, medium-fine silica-quartz sand exhibited a bulging and sloughing failure without weeping at approximately $70 \%$ saturation. However, the other two materials exhibited an onset of weeping before failure due to larger pore space providing rapid drainage of the added water. The E57-60 material quickly fell under a load less than $1 \mathrm{kPa}$. Due to significant suction effects of the platey grain structure, the T101 samples held an average of about $10 \mathrm{kPa}$.

In the case of the S101 material, the specimens exhibited a non-uniformity in slumping. This suggests, as with the dry cases, that failure does not necessarily meet the simultaneous rupture criteria. Thus, in the case of Equation 1, the predicted strength would be in excess of the observed lack of shear resistance. Conversely, the E57-60 and T101 samples, due to their inability to continuously retain additional pore fluid, do exhibit a strength increase due to suction and a more simultaneous failure mode. However, the same unresolved query of accounting for the effects of suction twice still remains for these samples.

\section{Conclusions}

For sub-angular silica-quartz sand, it is suggested that the pore fluid had a lubrication (strength weakening) effect, and the assumption of structure stiffening (strength increase) from matric suction may not be valid at near-surface conditions. However, the effects of suction are very pronounced on the platey crystalline structure of the fused quartz sand and contribute to a large increase in strength between the dry and wet conditions and only a mild strength loss at the point of weeping.

\section{Acknowledgements}

The authors would like to acknowledge M.D. Antwine and W.R. Rowland for their assistance in conducting the experiments. Funding was provided by the ERDC Future Innovations Fund: Unlocking the physics of near-surface soil mechanics. Permission to publish was granted by the Director, Geotechnical and Structures Laboratory, U.S. Army Engineer Research and Development Center, with unlimited distribution.

\section{References}

1. Fannin, R. J., Eliadorani, A., \& Wilkinson, J. M. T. (2005). Geotechnique, 55(6), 467-478.

2. Huang, Y., Cheng, H., Osada, T., Hosoya, A., \& Zhang, F. (2015). J. of Geotech. and Geoenviron. Eng, 06015005-1-06015005-6.

3. Lancelot, L., Shahrour, I., \& Al Mahmoud, M. (2006). J. of Eng. Mech., 132(12), 1396-1399.

4. Han, Z., \& Vanapalli, S.K. (2016). Geotechnique 66(8), 627-647.

5. Terzaghi, K., Peck, R.B, \& Mesri, G. (1996). Soil Mechanics in Engineering Practice. New York, NY, USA: John Wiley \& Sons.

6. Taylor, O. D. S., Winters, K. E., Berry, W. W., Walshire, L. A., \& Kinnebrew, P. G. (2019). Canadian Geotechnical Journal, 56(3), 307-319.

7. Ezzein, F. M. and Bathurst, R. J., 2011, Geotech. Test. J., Vol. 34, No. 6, pp. 1-8.

8. Peters, S. B., Siemens, G., \& Take, W. A. (2011). Geotechnical Testing Journal, 34(5), 445-456

9. Siemens, G. A., Peters, S. B., \& Take, W. A. (2013). Water Resources Research, 49(2), 851-863.

10. Siemens, G. A., Mumford, K. G., \& Kucharczuk, D. (2015). Geotechnical Testing Journal, 38(5), 620630.

11. Taylor, O.-D.S., Berry, W.W., Winters, K.E., Rowland, W.R., Antwine, M.D., and Cunningham, A.L. (2017). Geotechnical Testing Journal 40(2): 284-301.

12. Winters, K.E., Taylor, O.-D.S., Berry, W.W, Rowland, W.R., Antwine, M.D., \& Cunningham, A.L. (2016). ASCE GeoChicago 2016. 